\title{
Relever le défi de la diversité : une comparaison des idéologies en éducation en contexte minoritaire et majoritaire au Nouveau-Brunswick et en Saskatchewan Meeting the Challenge of Diversity: A Comparison of Ideologies in Education in a Minority and Majority Context in New Brunswick and Saskatchewan \\ Cotejar los retos de la diversidad: una comparación de las ideologías en educación en contextos minoritarios y mayoritarios en Nuevo Brunswick y en Saskatchewan
}

\author{
Nicole Gallant et Wilfrid B. Denis
}

Volume 36, numéro 1, printemps 2008

Rapports ethniques et éducation : perspectives nationales et internationales

URI : https://id.erudit.org/iderudit/018094ar

DOI : https://doi.org/10.7202/018094ar

Aller au sommaire du numéro

Éditeur(s)

Association canadienne d'éducation de langue française

ISSN

0849-1089 (imprimé)

1916-8659 (numérique)

Découvrir la revue

\section{Résumé de l'article}

En dépit d'efforts récents par les gouvernements fédéral et provinciaux et par le milieu associatif pour augmenter le nombre d'immigrants, les communautés francophones en milieu minoritaire au Canada en reçoivent très peu. En conséquence, très peu de programmes et de services particuliers existent pour les quelques élèves issus de l'immigration. Les défis sont particulièrement aigus pour les provinces où l'immigration est faible même du côté majoritaire. Dans cet article, nous faisons un inventaire des politiques ministérielles et des programmes scolaires touchant l'accueil et l'intégration des élèves immigrants, du côté anglophone en comparaison avec le côté francophone en

Saskatchewan et au Nouveau-Brunswick. Nous constatons tout d'abord une certaine disparité entre les anglophones et les francophones, mais aussi un manque d'harmonisation entre les diverses mesures, qui demeurent généralement à un stade expérimental. Globalement, ces mesures se situent dans une approche idéologique de type multiculturaliste participative, c'est-à-dire où on tient compte des différences portées par les immigrants, mais surtout pour leur permettre individuellement de rejoindre les autres élèves. Toutefois, le manque d'encadrement de ces mesures et l'absence de programmes créent d'importantes disparités entre les écoles, même sur le plan de l'approche idéologique.

Citer cet article

Gallant, N. \& Denis, W. B. (2008). Relever le défi de la diversité : une comparaison des idéologies en éducation en contexte minoritaire et majoritaire au Nouveau-Brunswick et en Saskatchewan. Éducation et francophonie, 36(1), 142-160. https://doi.org/10.7202/018094ar

Tous droits réservés (C) Association canadienne d'éducation de langue française, 2008 cest protége par la loi sur le droit d'auteur. L'utilisation des services d’Érudit (y compris la reproduction) est assujettie à sa politique d'utilisation que vous pouvez consulter en ligne. 


\section{Relever le défi de la diversité :}

une comparaison des idéologies en éducation en contexte minoritaire et majoritaire au

\section{Nouveau-Brunswick et en Saskatchewan}

\section{Nicole GALLANT}

Professeure-chercheure, Institut national de la recherche scientifique (INRS) Urbanisation, culture et société, Québec, Canada

Wilfrid B. DENIS

Sociologue, Université de la Saskatchewan, Saskatchewan, Canada

\section{RÉSUMÉ}

En dépit d'efforts récents par les gouvernements fédéral et provinciaux et par le milieu associatif pour augmenter le nombre d'immigrants, les communautés francophones en milieu minoritaire au Canada en reçoivent très peu. En conséquence, très peu de programmes et de services particuliers existent pour les quelques élèves issus de l'immigration. Les défis sont particulièrement aigus pour les provinces où 
l'immigration est faible même du côté majoritaire. Dans cet article, nous faisons un inventaire des politiques ministérielles et des programmes scolaires touchant l'accueil et l'intégration des élèves immigrants, du côté anglophone en comparaison avec le côté francophone en Saskatchewan et au Nouveau-Brunswick. Nous constatons tout d'abord une certaine disparité entre les anglophones et les francophones, mais aussi un manque d'harmonisation entre les diverses mesures, qui demeurent généralement à un stade expérimental. Globalement, ces mesures se situent dans une approche idéologique de type multiculturaliste participative, c'est-à-dire où on tient compte des différences portées par les immigrants, mais surtout pour leur permettre individuellement de rejoindre les autres élèves. Toutefois, le manque d'encadrement de ces mesures et l'absence de programmes créent d'importantes disparités entre les écoles, même sur le plan de l’approche idéologique.

\section{ABSTRACT}

\section{Meeting the Challenge of Diversity: A Comparison of Ideologies in Education in a Minority and Majority Context in New Brunswick and Saskatchewan}

Nicole GALLANT

INRS - Urbanization, Culture and Society, Quebec, Canada

Wilfrid B. DENIS

University of Saskatchewan, Saskatchewan, Canada

Despite recent efforts by federal and provincial governments and the community milieu to increase the number of immigrants, French speaking sectors in Canadian minority areas receive very few. Consequently, few programs and specific services exist for the small number of immigrant students. The challenges are particularly acute for provinces with low immigration levels, even on the majority side. In this article, we make an inventory of ministerial policies and school programs in Saskatchewan and New-Brunswick geared to welcoming and integrating immigrant students in the English-speaking schools compared to the French-speaking schools. First of all, we notice a certain discrepancy between Anglophone and Francophone schools, along with a lack of harmonization among the various measures, which generally remain at an experimental stage. Overall, these measures lie in a participative and multiculturalist ideological approach, which take the immigrants' differences into account and above all, allows them to reach out individually to other students. However, the lack of supervision for these measures and the absence of programs create considerable discrepancies among schools, even in terms of the ideological approach. 


\section{RESUMEN}

\section{Cotejar los retos de la diversidad: una comparación de las ideologías en educación en contextos minoritarios y mayoritarios en Nuevo Brunswick y en Saskatchewan}

Nicole GALLANT

INRS - Urbanización, cultura y sociedad, Quebec, Canadá

Wilfrid B. DENIS

Universidad de Saskatchewan, Saskatchewan, Canadá

A pesar de los recientes esfuerzos de los gobiernos federal y provincial y del medio asociativo tratando de aumentar el número de inmigrantes, las comunidades francófonas en medio minoritario en Canadá acogen muy pocos inmigrantes. Lógicamente, existen pocos programas y servicios particulares para los alumnos provenientes de la inmigración. Los desafíos son particularmente agudos en las provincias en donde la inmigración es débil, incluso del lado mayoritario. En este artículo, hemos inventariado las políticas ministeriales y los programas escolares relacionados con la recepción y la integración de alumnos inmigrantes, comparando el lado anglófono con el lado francófono en Saskatchewan y en Nuevo Brunswick. Por principio hemos constatado una cierta disparidad entre anglófonos y francófonos, así como la ausencia de armonización entre las diversas medidas, que invariablemente quedan en un estadio experimental. Globalmente, dichas medidas se sitúan al interior de un enfoque ideológico de tipo multicultural participativo, en el cual se toman en cuenta las diferencias propias de los inmigrantes, sobre todo con el fin de permitir a los alumnos de emparejarse con el resto del grupo. Sin embargo, la ausencia de directivas y de programas para dichas medidas, provocan disparidades importantes entre las escuelas, incluso en lo concerniente al enfoque ideológico.

\section{Introduction}

Les communautés francophones en milieu minoritaire au Canada attirent très peu d'immigrants, en raison de deux grandes tendances. Premièrement, c'est un fait reconnu que les immigrants au Canada s'établissent surtout dans les grandes villes. Montréal, Toronto et Vancouver reçoivent à elles seules 80 \% des immigrants, tandis qu'un autre $10 \%$ s'installe à Calgary, Edmonton et dans la région d'Ottawa-Gatineau (Roy, Belkhodja et Gallant, 2007: 87). Le Nouveau-Brunswick et la Saskatchewan accueillent moins de $1 \%$ de l'immigration totale au Canada (Garcea, 2007). Entre 1997 et 2004, le Nouveau-Brunswick a reçu entre 600 et 800 immigrants par année pour 729500 habitants en 2001. Ce chiffre a augmenté de 1000 à 1600 entre 2005 et 
2006. Durant la même période, la Saskatchewan en a accueilli entre 1700 et 1900 pour une population de 978900 en 2001 et ce chiffre a augmenté de 2000 à 2700 entre 2005 et 2006 (Immigration Canada, 2007). Les deux tiers des arrivants dans ces provinces s'installent dans les villes de Saint-Jean, Fredericton et maintenant Moncton au Nouveau-Brunswick, et de Regina et Saskatoon en Saskatchewan.

La deuxième tendance importante est que les immigrants francophones s'établissent habituellement au Québec. En effet, seulement $3 \%$ du total d'immigrants francophones au Canada s'installent hors Québec; ces deux provinces en reçoivent donc très peu (Quell, 2002). En plus, environ la moitié des immigrants francophones qui s'installent à l'ouest du Québec effectuent un transfert linguistique vers l'anglais comme langue d'usage (Jedwab, 2001:61). Comme pour les francophones en milieu minoritaire, ce transfert linguistique dépend en grande partie de la faiblesse de l'appui institutionnel, qui est doublement problématique pour les nouveaux arrivants. En effet, les institutions manquent tant du côté des services reliés à l'immigration que du côté des services en français. De surcroît, les tendances de mobilité géographique des immigrants francophones suivent de près celles de l'ensemble des immigrants, de sorte que la grande majorité entreprend une deuxième migration vers les villes de Toronto, Vancouver et Montréal (Jedwab, 2001).

Pour ces deux raisons, les communautés francophones en milieu minoritaire sont peu habituées à l'immigration et demeurent donc relativement homogènes sur le plan ethnoculturel. L'intégration des immigrants dans ces communautés relève donc de dynamiques assez particulières. Notre étude porte sur les francophones minoritaires du Nouveau-Brunswick et de la Saskatchewan, ce qui permet de montrer tant des distinctions provinciales que des constantes vraisemblablement attribuables au contexte francophone minoritaire.

Notre analyse comparative des politiques ministérielles et des programmes scolaires favorisant l'accueil et l'intégration des élèves immigrants de ces deux provinces permet d'identifier l'approche idéologique prédominante des institutions éducatives tant de la majorité que de la minorité linguistique. Les pages suivantes expliqueront notre approche théorique et méthodologique avant de présenter des éléments de contexte propres aux deux provinces à l'étude. Puis, nous décrirons l'intersection de l'immigration et de l'éducation telle que reflétée dans les politiques et mesures existantes, ou absentes, aux niveaux des ministères et des secteurs éducatifs anglophone et francophone. L'analyse terminera avec des pistes d'amélioration de la situation.

\section{Problématique et méthodologie}

\section{Éléments théoriques}

Plusieurs études ont dégagé les étapes successives des développements idéologiques des politiques d'immigration au Canada (Knowles, 1997). Au départ, ces études concernaient surtout les critères d'admission des immigrants, mais analysent de plus en plus une multitude de facettes du phénomène, notamment l'accès au marché du travail, le logement, et même des aspects plus symboliques. Notre étude 
porte sur le système d'éducation et la façon dont s'opèrent l'accueil et l'intégration des enfants immigrants dans les écoles ${ }^{1}$ surtout d'après les idéologies latentes révélées par les stratégies retenues par les gouvernements.

Ces idéologies latentes se rattachent aux grands courants idéologiques nationaux et sont perceptibles dans les politiques concernant la diversité ethnoculturelle du Canada (Fleras et Elliott, 2007: 324-331). La première approche, qui a dominé dès les débuts du Canada jusqu'aux années soixante, fut celle du monoculturalisme ou de l'anglo-conformité. Dans ce contexte idéologique, l'école présentait alors un modèle unique de société, celui de la culture anglo-dominante au Canada, auquel les immigrants devaient se conformer. Par la suite, la montée du multiculturalisme a pris différentes formes avec l'adoption par le gouvernement fédéral d'une politique sur le multiculturalisme en 1971, jusqu'à la loi sur le multiculturalisme en 1988. Cette évolution législative et la réponse des diverses communautés minoritaires affectées ont amené l'idéologie du multiculturalisme à changer graduellement d'une approche de célébration des différences ethniques à la recherche d'égalité. Dans les écoles, ces changements ont donné lieu à trois modèles pédagogiques distincts (Fleras et Elliott, $2007: 324-331$ ), qui peuvent exister chacun seul ou coexister dans un même système scolaire. Le premier modèle, celui de l'enrichissement, est offert à tous les élèves; le programme d'études régulier est enrichi d'informations sur diverses cultures afin de faire connaître et apprécier la diversité culturelle. Le deuxième modèle est celui de la conscientisation (enlightenment) qui vise non seulement l'enrichissement, mais aussi le changement d'attitude et la reconnaissance des inégalités entre les groupes culturels. En plus de connaissances objectives sur les différences culturelles, les élèves sont sensibilisés aux rapports majoritaires-minoritaires et aux phénomènes de victimisation ou de marginalisation de certains groupes culturels. Le troisième modèle est celui de l'épanouissement, de la participation ou de l'autonomisation (empowerment) ${ }^{2}$ par lequel des espaces sont créés afin d'offrir une atmosphère sécuritaire pour les élèves de communautés minoritaires. De plus, des efforts additionnels sont faits pour embaucher des professeurs issus de communautés minoritaires qui deviennent des modèles pour ces élèves ${ }^{3}$.

Finalement, depuis quelques années, une quatrième approche se manifeste, celle d'un multiculturalisme civique qui inclut la participation à toutes les institutions canadiennes, l'identification comme Canadien et le sentiment d'être citoyen à part entière (Fleras et Elliott, 2007 : 284-285). Cette participation globale à la société

1. Une étude similaire au Nouveau-Brunswick, en 2004, avait démontré de surcroît la façon dont le discours officiel du ministère, des commissions scolaires et des écoles présentait la question de la diversité et de l'identité (Gallant, 2006).

2. Le terme anglais empowerment est traduit habituellement par autonomisation. Parfois le terme habilitation est aussi utilisé. Il nous semble que ces mots ne sont pas entièrement fidèles aux différentes nuances comprises dans empowerment. En anglais, le terme s'applique aux individus qui atteignent un certain niveau d'autonomie et de contrôle sur leur vie ainsi qu'à des groupes ou des collectivités qui réussissent à établir un certain degré d'autonomie collective et d'autodétermination. Nous avons du mal à traduire l'envers d'autonomie qu'est le disempowerment.

3. Cette approche peut s'étendre jusqu'à la création d'écoles minoritaires pour desservir des groupes distincts comme les Afro-américains ou les autochtones. Les écoles de "français langue première » en contexte minoritaire cadrent dans ce modèle également, tout en y ajoutant, dans certaines provinces, la dimension de contrôle direct par les parents francophones. 
canadienne exige une prise de conscience des dimensions multiples de la discrimination raciale dans la plupart des institutions canadiennes. En éducation, on préconise davantage une approche antiraciste qui s'attaque directement aux structures sociales inégales afin d'enrayer le racisme et l'intolérance. Ainsi, le contenu pédagogique aborde la valeur de la diversité dans une perspective d'égalité, de justice et de redistribution du pouvoir. Ces questions, lorsque soulevées à l'intérieur du système éducatif, doivent sensibiliser les participants, autant le personnel enseignant que les élèves, au besoin de changements structuraux tant dans le système scolaire que dans les autres institutions de la société (Fleras et Elliott, 2007 : 284-285).

Ces approches correspondent aux quatre grands modèles identifiés par Poirier (2005) quant à l'intégration des immigrants dans des systèmes quelconques. D'abord, l'assimilationnisme radical se rattache au monoculturalisme; ensuite, l'universalisme civique (ou intégrationnisme), où «les différences (choix moraux, croyances religieuses, comportements, goûts) ne sont pas niées mais confinées à l'espace privé" alors qu'on attend des immigrants qu'ils adoptent les valeurs civiques et politiques de la société d'accueil. Le troisième, le multiculturalisme, valorise la diversité culturelle comme une richesse, dont les politiques doivent tenir compte, ou qu'elles devraient même soutenir; et enfin le quatrième, l'interculturalisme, tente de faire un amalgame entre l'universalisme civique et le multiculturalisme, où on cherche «la construction de références communes fortes", qui aident à former un espace commun permettant la rencontre des cultures, car l'on estime que «l'immigrant et la société d'accueil doivent tous les deux s'adapter l'un à l'autre ».

\section{Méthodologie}

Lesquelles de ces orientations idéologiques se manifestent dans les approches utilisées dans les écoles francophones des deux provinces à l'étude? Pour répondre à cette question, nous avons adopté une approche plus sociopolitique que pédagogique de l'école, c'est-à-dire en considérant l'école en tant qu'agent de socialisation de la prochaine génération. Contrairement à la plupart des autres agents de socialisation des jeunes, comme les parents et les amis, l'école est un agent de socialisation formel contrôlé par l'État. Les politiques éducatives révèlent donc l'idéologie des autorités politiques. C'est pourquoi cette première étude exclut les contenus pédagogiques des programmes au profit d'un examen de l'idéologie sous-jacente aux mesures et structures pertinentes des institutions scolaires.

Dans un premier temps, nous examinerons les programmes administratifs et les mesures relevant des ministères de l'Éducation des deux provinces, afin d'inventorier les directives générales et les mesures existantes concernant les immigrants et la diversité. Ensuite, nous nous pencherons plus spécifiquement sur ce qui existe d'abord du côté anglophone et ensuite du côté francophone. Pour cerner les idéologies qui influencent les politiques et les programmes d'accueil et d'intégration des immigrants en éducation, nous avons analysé les documents et les sites web pertinents des ministères de l'Éducation et des divisions scolaires. Comme nous le verrons, très peu de programmes existent. Nous avons aussi rassemblé les énoncés 
d'objectifs et de missions des divisions scolaires et d'écoles francophones, afin de voir si la diversité était mentionnée ou comment la clientèle cible était présentée.

Dans un second temps, nous présenterons une analyse documentaire qualitative de ce matériel. La rareté du matériel ne justifiait pas une analyse quantitative. Notre analyse comporte deux volets. Le premier relève d'une technique assez classique de l'analyse du discours, soit l'étude de l'énonciation. Nous nous sommes intéressés à la façon dont les groupes étaient désignés, en particulier dans le but de cerner les conceptions exprimées de la diversité interne, ou de son absence. Le second et principal volet de l'analyse consiste à interpréter la façon dont les politiques mises en œuvre présentent l'insertion de l'élève immigrant, avec un intérêt particulier sur ce que cela révèle comme attentes par rapport au résultat final, en termes de conceptions de la diversité ethnoculturelle. Ces types d'attentes sont classés selon les catégories décrites précédemment, développées par Fleras et Elliott, ainsi que, dans une moindre mesure, celles de Poirier.

En terminant, mais de manière parfois concomitante à nos analyses du premier et second volets, nous avons confirmé l'orientation et la mise en application de ces politiques par des entrevues téléphoniques ou en personne avec les personnes responsables de ces programmes. Cette démarche a également permis de compléter l'inventaire des mesures ou programmes administratifs existants. Ces employés des ministères et des divisions scolaires étaient informés de la nature de notre recherche; nous les avons surtout invités à décrire les mesures entreprises, mais aussi à discuter des objectifs sous-jacents, afin de corroborer nos interprétations. Peu de personnes s'occupent de tels programmes; nous nous sommes limités aux répondants clés dans les deux provinces, soit trois ou quatre personnes par province.

\section{Contexte}

\section{L'immigration au Nouveau-Brunswick et en Saskatchewan}

La Saskatchewan et le Nouveau Brunswick figurent parmi les six provinces qui, en 2007, géraient l'immigration par le biais d'ententes fédérales-provinciales. Les premières ententes furent signées respectivement en 1998 pour la Saskatchewan et en 1999 pour le Nouveau-Brunswick, puis renouvelées ou refaites par la suite (Bourgeois et al., 2006). Au Nouveau-Brunswick, le «programme des candidats » relevait jusqu'à tout récemment du ministère Entreprises Nouveau-Brunswick, mais, en 2007, une nouvelle politique plus englobante a créé un Secrétariat à la repopulation avec deux volets, soit le rapatriement et l'immigration ${ }^{4}$.

Ces deux provinces n'ont donc pas une longue expérience de l'accueil des immigrants, et leur communauté francophone encore moins. Les chiffres donnés précédemment en témoignent. La communauté fransaskoise a exploré certains aspects de la diversité dans sa Commission sur l'inclusion (Denis et al., 2006), mais on ne peut

4. Cette nouvelle politique était en préparation depuis quelques années mais un changement de gouvernement en octobre 2006 a considérablement retardé le processus en le réorientant vers une approche plus globale. 
pas dire que des services ou des structures d'accueil existent. Ainsi, l'accueil et l'intégration des immigrants sont laissés à des initiatives ponctuelles qui varient considérablement d'un immigrant à un autre (Gallant, Roy et Belkhodja, 2007). Du côté du Nouveau-Brunswick, les francophones se sont dotés de quelques structures d'accueil et d'intégration des immigrants, en particulier le Carrefour d'immigration rurale (CIR) à Saint-Léonard, dans le nord-ouest de la province, et plus récemment le Centre d'accueil et d'intégration des immigrants du Moncton métropolitain (CAIIMM), qui offre des services spécifiquement en français à Moncton ${ }^{5}$. De plus, une réflexion plus englobante et systématique est en cours depuis plusieurs années sous l'égide de la Table de concertation sur l'immigration francophone au NouveauBrunswick. Cette table réunit la Société des Acadiens et Acadiennes du NouveauBrunswick, des représentants des gouvernements provincial et fédéral, divers organismes francophones, et quelques chercheurs du Centre Métropolis Atlantique, mais parmi les quelques initiatives concrètes, aucune n'est liée directement au secteur de l'éducation.

\section{Le système éducatif au Nouveau-Brunswick et en Saskatchewan}

Pour comprendre la structure et l'orientation des programmes d'accueil scolaire, il est important de situer le contexte du système scolaire francophone de chacune des provinces, car elles diffèrent grandement à ce chapitre. En effet, au NouveauBrunswick, le ministère de l'Éducation est entièrement dédoublé à partir du sousministre pour ce qui a trait au programme et au curriculum, et ce, depuis 1974 (Basque, Barrieau et Côté, 1999: p. 96), reflétant ainsi la dualité linguistique de la province $^{6}$. En outre, le système scolaire est structuré selon deux cartes géographiques répartissant les conseils scolaires francophones et anglophones et les régions administratives sur l'ensemble de la province, en deux systèmes indépendants l'un de l'autre. En conséquence, une région donnée est régie aussi bien par un conseil scolaire francophone qu'un conseil scolaire anglophone, qui dirigent chacun leurs propres écoles et qui s'étendent sur des territoires différents. Les districts francophones sont moins nombreux en raison des effectifs plus limités et sont divisés selon des frontières différentes qui ne recoupent qu'occasionnellement celles des districts anglophones. Dans ce contexte, le système scolaire francophone au NouveauBrunswick dispose d'une certaine autonomie institutionnelle, qui pourrait lui permettre de développer des programmes relevant d'une approche idéologique propre relativement à l'intégration des immigrants.

En revanche, du côté de la Saskatchewan, l'autonomie institutionnelle des écoles francophones est moindre, dans la mesure où le regroupement le plus élevé se situe au niveau de la division scolaire plutôt qu'au niveau ministériel. En effet, en

5. Il existait bien un autre organisme desservant les immigrants francophones de la région, mais il s'agissait d'un organisme bilingue à prédominance anglophone.

6. Les francophones du Nouveau-Brunswick représentent un tiers de la population provinciale. Cette dualité démographique s'est traduite au niveau des structures politiques par l'établissement de la dualité linguistique au niveau gouvernemental (Loi sur les langues officielles de 1969; Loi 88, reconnaissant l'égalité des deux communautés linguistiques du Nouveau-Brunswick) (Bourgeois et al., 2006). 
1993, les Fransaskois obtenaient la gestion de leurs écoles par la refonte de l'Acte scolaire (Denis, 2006). Au début, cette refonte établissait huit divisions scolaires, une pour chaque école, mais celles-ci se sont toutes regroupées sous l'égide d'une seule division scolaire en $1996^{7}$. La Division scolaire fransaskoise ( $\left.{ }^{\circ} 310\right)$ dessert toute la province et gère aujourd'hui douze écoles dans des communautés dispersées sur l'ensemble du territoire provincial. En plus de huit communautés rurales, ses écoles se retrouvent dans quatre villes : Regina, Saskatoon, Prince Albert et North Battleford. Les immigrants francophones s'établissent surtout dans ces villes, mais particulièrement à Regina.

Dans la plupart des communautés francophones en contexte minoritaire, les luttes pour obtenir le contrôle et la gestion scolaires furent tellement longues et pénibles que l'accent a été mis presque exclusivement sur ce qui unissait les communautés francophones (Behiels, 2004; Martel, 1991, 2002). Dans un tel contexte, il n'est pas surprenant que l'approche préconisée soit celle de la monoculture francophone, en contrepartie au système dominant dans une perspective de mesures de protection externes (Kymlicka, 1995). En effet, jusqu'à relativement récemment, les communautés francophones en situation minoritaire opposaient à l'idéologie dominante d'anglo-conformité leur idéologie, souvent latente et vraisemblablement inconsciente, de franco-conformité (Heller, 1998). Étant donné que ces groupes se perçoivent comme une minorité qui doit se protéger d'une majorité dominante, il leur faut un certain temps pour arriver à concevoir l'enjeu de la diversité interne à la communauté francophone minoritaire. Pourtant, à l'endroit de leurs immigrants, elles ont tendance à se comporter comme une majorité assimilatrice, même si le milieu associatif acadien et francophone minoritaire du Canada est de plus en plus sensibilisé à la question de l'immigration (Gallant et Belkhodja, 2005).

\section{Les ministères et le système majoritaire}

\section{Le cas du Nouveau-Brunswick}

Au Nouveau-Brunswick, le dédoublement du ministère entraîne des différences importantes au niveau du traitement de l'immigration selon la langue du secteur scolaire. En effet, des mesures et ressources existent depuis un certain temps dans le secteur anglophone en raison des nombres plus importants. Ainsi, on assiste au développement d'une véritable réflexion d'ensemble sur la diversité, réflexion qui passe généralement par l'enseignement de la langue seconde. Concrètement, le ministère a développé un document cadre (1996), qui dépasse largement les simples principes de l'apprentissage d'une langue nouvelle, puisqu'il fait aussi état des étapes d'adaptation culturelle et autres du migrant. De fait, plusieurs études montrent bien

7. À cette époque, la Saskatchewan comptait le plus grand nombre de divisons scolaires au Canada. Le regroupement des écoles francophones s'est fait à l'instigation des communautés fransaskoises, offrant ainsi un modèle d'intégration administrative aux divisions scolaires anglophones. En plusieurs étapes, le nombre de commissions scolaires en Saskatchewan passa d'un total de 99 en 2000 à 28 en 2005-2006. 
que les besoins des élèves immigrants et réfugiés dépassent de loin les questions d'apprentissage de l'anglais (Epstein, 2006; MacKay, 2005; Manitoba, 2006). Ce nouveau cadre manifeste une approche multiculturaliste en reconnaissant les différences et leurs effets sur l'adaptation des immigrants. Mais aucun élément ne concerne le contenu pédagogique destiné à l'ensemble des élèves et, en ce sens, il ne peut s'agir, dans la typologie de Fleras et Elliott, ni du modèle d'enrichissement ni du modèle de conscientisation. Toutefois, on y retrouve certains éléments de type «participation", puisqu'on cherche explicitement à ce que l'élève immigrant arrive à participer pleinement à la société en tant que futur citoyen canadien. Par exemple, on peut lire dans ce document que «all school personnel must be aware of cultural differences and be sensitive to difficulties of cultural adjustments that play a significant role in learning English as a second language» (NB-ESB, 1996: 1). Ce document, qui sert pourtant de pilier à l'approche gouvernementale en matière d'insertion des immigrants dans le système scolaire, en est un des rares au ministère à ne pas être disponible dans les deux langues (en dehors des descriptions de programmes, qui diffèrent évidemment d'un secteur à l'autre).

Plus récemment, au printemps 2007, le ministre de l'Éducation Lamrock a annoncé un plan d'action qui comporte un bref volet destiné aux immigrants, mais qui porte surtout sur l'apprentissage de la langue seconde, donc sur les immigrants allophones (Ministère de l'Éducation du Nouveau-Brunswick, 2007: 9, 22 et 26). La question de la diversité ethnoculturelle n'est pas traitée. Comme il s'applique aux deux secteurs, ce document constitue la première initiative formelle à toucher explicitement le secteur francophone.

\section{Le cas de la Saskatchewan}

La Saskatchewan est à revoir ses politiques et son orientation pédagogique pour l'ensemble de son système éducatif. Les analyses que nous proposons ne sont donc valides que pour la période se terminant à l'été 2007. Nos analyses devront être mises à jour lorsque ces nouvelles politiques apparaîtront. La Saskatchewan semble sur papier plus avancée que le Nouveau-Brunswick, car le ministère de l'Apprentissage a adopté sa propre politique officielle sur le multiculturalisme en 1994. Le discours de cette politique établie à la suite des recommandations d'un groupe de travail sur le multiculturalisme (1989) ressemble de près à celui de la politique provinciale sur le multiculturalisme (1974). Les deux politiques sont souvent présentées ensemble. Sa vision est celle «d'une société qui est fière de son héritage et qui encourage la collaboration entre le foyer, la communauté et l'école pour réaliser l'égalité d'accès et d'opportunité pour tous ». La politique vise à encourager «le respect et la valorisation de soi et des autres qui sont différents selon leur race, religion, statut ou attributs personnels ». Les objectifs incluent la connaissance et l'appréciation de la diversité culturelle et son impact positif sur l'histoire, l'économie et la culture du Canada, ainsi que les effets de «la polarisation ethnique, les tensions et les conflits, le racisme, la discrimination et les préjugés ». Un objectif pédagogique fait référence à l'autonomisation (empowerment) des élèves afin qu'ils s'engagent politiquement et socialement dans le changement social constructif et autodéterminant (notre traduction) 
(Sask Learning, 1994) ${ }^{8}$. Selon les modèles de Fleras et Elliott présentés précédemment, la politique sur le multiculturalisme offre un mélange des modèles d'enrichissement et de conscientisation et une référence à l'autonomisation. L'éducation antiraciste n'est pas abordée. Mais pour prendre corps, les trois autres modèles dépendent surtout de l'application de la politique et des ressources que le ministère souhaite y consacrer. Ces ressources semblent insuffisantes.

Par ailleurs, le ministère se préoccupe depuis longtemps de questions d'équité. En effet, principalement à partir de 1980, divers documents traitent des questions de stéréotypes présents dans le matériel pédagogique et dans l'enseignement et du besoin de traitement équitable des élèves par rapport à leur orientation sexuelle, aux femmes, aux minorités visibles et aux autochtones. Dans ce cas, on pourrait parler d'une sensibilité antiraciste, bien que celle-ci ne se situe pas au niveau des contenus pédagogiques. Toutefois, l'accent porte souvent davantage sur les droits des femmes et des homosexuels que sur la diversité ethnoculturelle ${ }^{9}$.

Par contre, ces discours ne se traduisent pas toujours de façon directe par des gestes et des actions du ministère. En effet, la politique du multiculturalisme n'a pas véritablement été mise en œuvre et aucune ressource spécifique ne lui a été attribuée de sorte qu'elle demeure au niveau du discours. Dans les faits, aucune unité d'éducation multiculturelle n'a vu le jour et aucune mesure ministérielle n’a été adoptée. En conséquence, l'application de la politique est en fait déléguée aux commissions scolaires, avec comme résultat que les mesures, lorsqu'il y en a, varient grandement d'un contexte à l'autre. En l'absence de lignes directrices concrètes, c'est purement le fruit du hasard si un nouvel élève immigrant bénéficie de mesures répondant à ses besoins particuliers. Sans règlements clairs ou de programmation détaillée de la part du ministère, les commissions scolaires ont été prises complètement au dépourvu par l'arrivée d'élèves immigrants. L'approche en est une d'expérimentation, de projets ponctuels et de résolution de crises. La question d'accueil et d'intégration des élèves immigrants est presque toujours réduite à une question de l'apprentissage rapide de l'anglais afin d'arriver à une intégration dans les classes régulières.

Le gouvernement provincial alloue de l'argent spécifiquement pour l'enseignement de l'anglais langue seconde, mais ces montants sont inclus parmi d'autres «facteurs de vulnérabilité » et disparaissent dans l'ensemble du budget de chaque commission, sans directives concrètes quant aux services à livrer ${ }^{10}$. En conséquence, les coûts associés à l'insertion des nouveaux arrivants ont tendance à disparaître

8. La politique de 1994 inclut également une autre politique sur l'enseignement des langues traditionnelles (heritage languages) et explique que l'enseignement du français et des langues autochtones est la responsabilité d'autres unités du ministère, tel que le Bureau de la minorité de langue officielle pour le français.

9. C'est le cas par exemple de ce qui est placé sous l'onglet « Respect de la diversité » (Respect for diversity) du site Internet du ministère de I'Apprentissage (Saskatchewan Learning, 2004).

10. Dans le manuel de financement de l'éducation de la province, les coûts de l'enseignement de l'anglais langue seconde sont inclus dans un nouveau « facteur de vulnérabilité » qui englobe ces coûts parmi ceux des thérapeutes, de l'orthopédagogie, des psychologues, des soins infirmiers et des travailleurs sociaux, pour l'ensemble de chaque division scolaire. Le facteur de vulnérabilité est déterminé par quatre facteurs, soit le pourcentage, pour chaque division scolaire, de familles sous le seuil de la pauvreté, de familles monoparentales, de familles sous-scolarisées et de familles pour qui l'anglais n'est pas la première langue (Saskatchewan Learning, 2007: 8). 
dans le budget général des divisions scolaires tandis que le ministère a l'impression d'avoir tenu compte de ces coûts dans sa formule de financement. Sans une formule plus claire, l'allocation des ressources par les commissions scolaires est complètement aléatoire et dépend entièrement des priorités des commissions.

Certaines commissions scolaires anglophones désignent une ou deux écoles offrant des programmes plus intensifs. Mais la plupart des commissions se fient à des "enseignants d'anglais langue seconde» itinérants qui circulent d'école en école pour offrir à chaque élève immigrant quelques heures par semaine d'enseignement individualisé. Le reste du temps, ces élèves sont soumis à une approche d'immersion totale dans une classe régulière où ils opèrent dans une langue qu'ils ne maîtrisent pas. Souvent les élèves de la maternelle et de la première année ne reçoivent rien d'autre que l'approche d'immersion dans la plupart des commissions scolaires anglophones. Les niveaux suivants ne reçoivent habituellement que quelques heures d'anglais par semaine durant la première année post-migratoire.

Avec un tel système, il y a une grande lacune de ressources pédagogiques, d'aides-enseignants et de matériel d'appui soit dans chaque classe, soit pour chaque nouvel arrivant qui en a besoin. Pourtant, des recherches démontrent qu'un apprenant d'une autre langue première a besoin d'un an ou deux pour développer une compétence élémentaire en communication (basic interpersonal communicative skills ou BICS) et de cinq à sept ans pour atteindre un niveau de capacité cognitive linguistique approprié à son âge afin de pouvoir décoder le langage abstrait et décontextualisé (cognitive academic language proficiency ou CALP) (Epstein, 2006: 30-31).

En raison de leur diversité, il est difficile de classer ces mesures dans la typologie de Fleras et Elliott, mais il semble qu'elles cherchent toutes à tenir compte des différences sans nécessairement tenter de les amoindrir, de sorte qu'on peut parler d'une approche normative de type multiculturaliste. Encore ici, on semble viser surtout à améliorer les possibilités de participation des élèves immigrants, plutôt que la conscientisation des autres élèves mais en mettant l'accent sur l'adaptation du nouvel élève plutôt que sur les modifications de structures institutionnelles.

\section{Les mesures dans les écoles francophones}

Des programmes d'intégration des élèves immigrants existent donc dans les deux provinces du côté majoritaire, mais ils en sont encore à des stades de développement peu poussés, ne serait-ce qu'en raison des nombres. En revanche, du côté des francophones, il n'existe pas de programme commun, seulement des initiatives ponctuelles, qui sont embryonnaires. Il en résulte une importante disparité non seulement entre les immigrants francophones et les immigrants anglophones, mais aussi entre les immigrants francophones eux-mêmes. 


\section{Le discours du} secteur francophone relève d'une approche universaliste: dans cette optique, il ne doit pas y avoir de " programme spécifique ", car l'enfant immigrant doit être «traité comme les autres", " comme tout autre enfant», ayant «les mêmes droits et les mêmes services».

\section{Dans les écoles francophones du Nouveau-Brunswick}

En effet, du côté francophone du ministère, la situation est bien différente de celle du côté anglophone. Si le secteur anglophone a développé des ressources pour les élèves immigrants, surtout pour l'apprentissage de l'anglais comme langue seconde, pour sa part, le secteur francophone n'offre aucune ressource en français concernant les immigrants. Au contraire, le discours du secteur francophone relève d'une approche universaliste: dans cette optique, il ne doit pas y avoir de "programme spécifique», car l'enfant immigrant doit être "traité comme les autres", "comme tout autre enfant", ayant "les mêmes droits et les mêmes services " ${ }^{11}$. L'objectif semble être de ne pas faire de discrimination, mais il en résulte que les écoles françaises ne reçoivent du Ministère aucun soutien spécifique pour l'intégration des nouveaux arrivants. Ce discours insiste sur l'égalité des élèves immigrants, mais dans une perspective uniforme, universaliste, ce qui vise donc une égalité des droits, plutôt que des conditions. Dans cette optique, les différences culturelles et religieuses appartiennent à la sphère privée et les interventions politiques n'ont pas à en tenir compte. Or, il semble évident aujourd'hui que l'égalité juridique ou le traitement uniforme ne conduisent généralement pas directement à l'égalité réelle ${ }^{12}$. En ce sens, l'approche universaliste prônée par le secteur francophone, si elle était appliquée telle quelle, contraindrait les écoles à un comportement assimilateur.

Toutefois, en dépit du manque de ressources et d'encadrement ministériel, certaines écoles ont dû tenter de développer des mesures en raison de la demande, notamment dans les trois grandes régions urbaines de Saint-Jean, Fredericton et Moncton, qui attirent le plus grand nombre d'immigrants francophones. Le besoin est de plus en plus pressant car le nombre d'élèves immigrants récents a doublé dans la dernière année, passant de 77 en 2006 à environ 156 en mars 2007. Le district scolaire francophone responsable de ces régions est fermement engagé sur ce dossier et tente d'harmoniser diverses initiatives entreprises dans les écoles individuelles. Ce district a produit en avril 2005 une Trousse d'accueil et d'intégration des élèves immigrants nouvellement arrivés, qui est encore à l'étape de l'évaluation mais qui est néanmoins offerte comme ressource aux enseignants responsables d'élèves immigrants. Largement inspirée de documents similaires du Québec et, surtout, du Manitoba, cette trousse a été adaptée au contexte acadien et francophone minoritaire. On y traite aussi bien de l'accueil à l'école (on explique notamment comment réaliser la première rencontre et on suggère diverses activités possibles) que de l'intégration scolaire dans ses volets social, pédagogique et linguistique. Une courte section à la fin traite aussi de l'éducation interculturelle et de l'éducation à la citoyenneté.

Concrètement, les enfants immigrants sont immédiatement intégrés dans des classes régulières, car les effectifs ne justifient pas des classes d'accueil ${ }^{13}$. Toutefois,

11. Propos tenus au téléphone par divers membres du personnel du secteur francophone du ministère de l'Éducation et des conseils d'éducation de district, lorsqu'on leur demande de décrire les mesures existantes pour les immigrants.

12. Nous avons discuté ailleurs des raisons pour lesquelles un tel traitement d'égalité formelle des immigrants ne conduit justement pas à leur égalité réelle (Gallant, 2006).

13. Cette pratique a d'ailleurs été décriée par de nombreux auteurs. Voir notamment Patriciu et Laaroussi (2002). 
dans les quatre écoles ${ }^{14}$ qui reçoivent le plus d'immigrants, un enseignant est dégagé pendant une heure par jour afin d'agir comme responsable à l'accueil pour les élèves immigrants éparpillés dans diverses classes de différents niveaux. Cette personneressource rencontre ces élèves sur une base individuelle et en groupe afin de parfaire leur francisation, mais aussi de discuter librement de questions relatives à leur insertion scolaire et sociale.

Dans les écoles, l'approche consiste donc à tenir compte d'une différence, non pas spécifiquement ethnoculturelle ou religieuse, mais à tout le moins liée au parcours particulier de ces élèves. Les mesures adoptées cherchent à favoriser l'insertion scolaire et sociale individuelle, mais sans qu'un modèle très clair soit apparent: cherche-t-on à les mettre sur un pied d'égalité en vue de les assimiler ou cherche-ton à les intégrer au système scolaire tout en maintenant leur spécificité culturelle? Est-ce que cette spécificité culturelle est valorisée à l'intérieur de l'école ou est-elle reléguée au secteur privé? Il est impossible de répondre à ces questions car, en l'absence de directives claires en provenance du district, chaque enseignantressource y ira de sa propre interprétation des fonctions de ces mesures.

Toutefois, en juin 2006, ce district a aussi adopté en parallèle une politique qui spécifie le «mandat culturel, communautaire et identitaire» de l'école de langue française qui est de : «valoriser [...] la langue et la culture acadiennes et francophones" et « contribuer à la construction identitaire et la fierté acadienne et francophone des élèves» (DS1, 2006,1). Les expressions "acadienne» et "francophone" ne sont pas véritablement précisées; il n'est pas clair si les deux termes sont utilisés en juxtaposition pour marquer une différence ou s'ils sont synonymes. En fait, les deux termes sont mentionnés séparément en un seul endroit pour référer à une population «à majorité acadienne dans certains cas et minoritaire acadienne et francophone dans d'autres ». Ceci semblerait indiquer que, dans le deuxième cas, la population concernée n'est pas seulement acadienne ce qui sous-entend que le mot «francophone » est plus englobant que le mot «acadien». Sauf que le texte poursuit: « et auxquelles s'ajoutent plusieurs individus de diverses origines ayant choisi ces communautés comme lieu de résidence", ce qui implique que les immigrants ne sont pas inclus dans l'expression " acadienne et francophone $»^{15}$. Ainsi, tout en ayant une apparence d'ouverture à la diversité, cette politique n'est pas sans rappeler la situation idéologique observée en 2004 (Gallant, 2006), où les efforts pour exposer les élèves à l'existence de différentes cultures sur la planète constituent une exposition à la diversité mondiale... mais pas à la diversité locale. On y fait la promotion d'une fierté qui est celle du "patrimoine" et des origines, d'une certaine homogénéité interne à l'école face à une hétérogénéité extérieure au monde scolaire, ce qui prépare mal l'insertion sociale des élèves immigrants.

14. Une cinquième devait s'ajouter à compter de septembre 2007.

15. En fin de compte, il est possible que le qualificatif «francophone» ne soit pas là pour être inclusif, mais plutôt pour être restrictif en excluant des personnes d'origine acadienne qui ne parlent plus le français. 


\section{Dans les écoles francophones en Saskatchewan}

En ce qui a trait à la Saskatchewan, l'ensemble des écoles francophones de cette province relève aujourd'hui d'une seule et même commission scolaire. Sur le plan de l'accueil et de l'intégration des immigrants, celle-ci se trouve donc dans la même situation que les commissions scolaires anglophones de la province, c'est-à-dire qu'elle doit improviser ses mesures d'accueil sans autre financement que celui associé à l'apprentissage de la langue seconde fondu avec d'autres coûts. La Division scolaire fransaskoise commence son programme d'accueil seulement en $4^{\mathrm{e}}$ année, faute de ressources. Cette division a alloué, en 2006-2007, 20 \% de la tâche d'un enseignant pour une classe d'accueil dans une école ayant une trentaine d'élèves immigrants. Dans ses autres écoles, les élèves immigrants, moins nombreux, sont référés à l'orthopédagogue. Comme au Nouveau-Brunswick francophone, les mesures adoptées par cette commission se placent difficilement dans les modèles de Fleras et Elliott ou même dans ceux de Poirier.

\section{Conclusion}

Nous constatons que, tant au Nouveau-Brunswick qu'en Saskatchewan, très peu de programmes structurés existent pour l'intégration des immigrants, d'une part, et, d'autre part, que les écoles francophones sont nettement moins bien nanties

Dans ces deux provinces, des politiques ou des directives au niveau du ministère, accompagnées de ressources appropriées, tardent à venir. L'accueil concret et les mesures d'insertion scolaire et sociale dépendent de chaque commission scolaire, voire des écoles individuelles ou encore d'initiatives d'un enseignant isolé. de ce point de vue que les écoles anglophones. Toutes ces mesures, adoptées un peu au hasard et par expérimentation dans les deux provinces, sont-elles néanmoins adéquates? Notre objectif ici n'était pas d'en faire une évaluation détaillée, mais bien de faire une analyse des approches idéologiques qui se dissimulent derrière elles. Dans ces deux provinces, des politiques ou des directives au niveau du ministère, accompagnées de ressources appropriées, tardent à venir. En Saskatchewan, le ministère a établi une politique sur le multiculturalisme dans les écoles, mais sans y mettre les ressources pour une véritable mise en œuvre. Les fonds pour l'apprentissage du français ou de l'anglais comme langue seconde sont intégrés dans les budgets globaux des commissions scolaires. Au Nouveau-Brunswick, il n'y a pas de politique officielle du multiculturalisme ou de l'immigration au niveau du ministère de l'Éducation, mais quelques ressources sont disponibles pour l'apprentissage de la langue seconde. Dans les deux cas, c'est très insuffisant. L'accueil concret et les mesures d'insertion scolaire et sociale dépendent de chaque commission scolaire, voire des écoles individuelles ou encore d'initiatives d'un enseignant isolé ${ }^{16}$. Toutes ces mesures se situent dans le modèle participatif de Fleras et Elliott, mais dans une optique individuelle plutôt que globale. En somme, selon les typologies de Fleras et Elliott et de Poirier, les discours et les politiques des deux provinces reflètent une approche multiculturaliste assez classique et très peu influencée par les développements idéologiques récents.

16. Nous ne disposons pas ici de l'espace nécessaire pour traiter des besoins spécifiques des réfugiés, mais il est important de préciser qu'ils ne sont que rarement mentionnés de manière spécifique. 
Par ailleurs, l'absence de programmes et de mesures harmonisées entre les écoles s'explique sans doute par les faibles effectifs. Pourtant, si les efforts des deux provinces pour accroître le nombre d'immigrants portent leurs fruits, le nombre d'élèves immigrants va continuer de croître. Il est donc impératif que ces deux provinces se dotent d'outils adaptés pour favoriser l'accueil, l'intégration et l'insertion scolaire et sociale de ces enfants immigrants.

Ces outils devraient inclure :

- Des ressources financières spécifiques allouées à l'apprentissage d'une langue officielle, mais aussi à l'accueil et à l'insertion scolaire et sociale pour tous les élèves immigrants.

- Des guides, tels que ceux en anglais au Nouveau-Brunswick, pour tout le personnel, autant les enseignants de langue seconde que les autres enseignants en contact avec des élèves immigrants.

- Un guide simple sans être infantilisant destiné aux parents, portant sur le fonctionnement de l'école, les dates des congés fériés et autres fêtes soulignées à l'école, et les attentes générales envers l'élève et dans les occasions spéciales ${ }^{17}$.

- Pour l'ensemble des élèves, un programme pédagogique qui les expose à la diversité ethnoculturelle, non seulement dans le monde, mais aussi «ici», chez eux.

- Un mécanisme de coordination afin d'harmoniser les objectifs et les programmes fédéraux sur l'immigration, les programmes qui découlent des ententes fédérale-provinciales en immigration, et les politiques et programmes en éducation. Idéalement un tel mécanisme préconiserait une approche intégrée avec d'autres ministères comme la santé et les services sociaux.

Tous ces instruments doivent être harmonisés entre les différentes écoles, les divisions ou commissions scolaires et les ministères concernés. Ils doivent donc préalablement avoir fait l'objet d'une réflexion sérieuse quant à leurs objectifs et quant à l'approche idéologique générale à l'intérieur de laquelle on souhaite se situer.

17. Par exemple, ce n'est pas du tout évident qu'il faille apporter des petites cartes pour la Saint-Valentin ou que des vétérans ou du personnel militaire participeront aux cérémonies du Jour du souvenir, ce qui peut être troublant pour les réfugiés victimes de violence (MacKay, 2005). 


\section{Références bibliographiques}

BASQUE, M., N. Barrieau et S. Côté (1999). L'Acadie de l'Atlantique. Moncton : Centre d'études acadiennes.

BEHIELS, M. (2004). Canada's Francophone Minority Communities: Constitutional Renewal and the Winning of School Governance. Montreal: McGill-Queen's University Press.

BOURGEOIS, D. et W. Denis, D. Dennie, M. Johnson. (2006). La contribution des gouvernements provinciaux et territoriaux à l'épanouissement des communautés francophones en milieu minoritaire: un premier bilan et quelques prospectives. Moncton, N-B. : Université de Moncton, l'Institut canadien de recherche sur les minorités linguistiques et l'Institut canadien de recherche en administration et politiques publiques.

CUMMINS, J. (1988). From multicultural to anti-racist education: An analysis of programmes and policies in Ontario, dans Minority Education: From Shame to Struggle, sous la direction de Skutnabb-Kangas et Jim Cummins. Philadelphia: Multilingual Matters.

DENIS, W. (2006). Francophone Education in Saskatchewan: Resisting Anglo-Hegemony, dans A History of Education in Saskatchewan: Selected Readings, sous la direction de Brian Noonan. Regina: Canadian Plains Research Center, p. 87-108.

DENIS, W. et al. (2006). La commission sur l'inclusion dans la communauté fransaskoise : de la minorité à la citoyenneté. Rapport présenté au nom des sept commissaires à l'Assemblée communautaire fransaskoise. Regina, Sask.

DS1, District scolaire 01 (2006). Politique culturelle, communautaire et identitaire. http://www.district1.nbed.nb.ca/media_uploads/pdf/154.pdf (site consulté le 12 juin 2007).

EPSTEIN, R. (2006). "I walked a thousand miles before I met you... » Final Report of the Review of St. Paul's Roman Catholic Separate School Division. Saskatoon : St. Paul's Roman Catholic Separate School Division.

FLERAS, A. et Elliott, J. L. (2007). Unequal Relations: An Introduction to Race, Ethnic, and Aboriginal Dynamics in Canada. Toronto: Pearson.

GALLANT, N. (2006). L'école francophone du Nouveau-Brunswick face à la diversité, dans Enjeux et défis de l'immigration au Nouveau-Brunswick: Rendez-vous Immigration, sous la direction de Hélène Destrempes et Paul Ruggieri, p. 343-373.

GALLANT, N. et C. Belkhodja (2005). Production d'un discours sur l'immigration et la diversité par les organismes francophones et acadiens au Canada. Études ethniques canadiennes/Canadian Ethnic Studies, vol. 37, $\mathrm{n}^{\circ}$ 3, p.35-58. 
GALLANT, N., J.-O. Roy, C. Belkhodja (2007). L'immigration francophone en milieu minoritaire: portrait de quatre municipalités rurales. Revue des cantons de l'Est. À paraître.

GARCEA, J. (2007). Immigration to Smaller Communities in Saskatchewan. Nos diverses cités, numéro spécial «Collectivités rurales", sous la direction de Bill Reimer, $n^{0}$ 3. p. 146-152.

IMMIGRATION CANADA. (2007). Faits et chiffres. [En ligne] http://cic.gc.ca/francais/pub/faits2006/faits2006_provinces.html (site consulté le 12 juin 2007).

HELLER, M. (1998). Linguistic Minorities and Modernity: A Sociolinguistic Ethnography. New York: Longman.

JEDWAB, J. 2001. L'immigration et l'épanouissement des communautés de langue officielle au Canada: politiques, démographie et identité. Ministre des Travaux publics et Services gouvernementaux Canada.

KNOWLES, V. (1997). Strangers at our gates: Canadian immigration and immigration policy, 1540-1997. Toronto: Dundurn Press.

KYMLICKA, W. (1995). Multicultural Citizenship. A Liberal Theory of Minority Rights. Oxford: Clarendon Press.

MACKAY, T. et T. Tavares (2005). Building Hope: Appropriate Programming for Adolescent and Young Adult Newcomers of War Affected Backgrounds and Manitoba Schools - A Preliminary Report for Consultation and Discussion. Winnipeg: MN. Manitoba Education, Citizenship and Youth. Disponible en ligne: http://www.edu.gov.mb.ca/k12/cur/diversity/eal/building_hope.pdf (site consulté le 15 mai 2007).

MANITOBA EDUCATION, CITIZENSHIP AND TRAINING (2006). English as an Additional Language (EAL) and Literacy, Academics, and Language Kindergarten to Grade 12 (Ébauche pour consultation, 7 septembre 2006). Disponible en ligne : http://www.edu.gov.mb.ca/k12/cur/diversity/eal/framework/eal.pdf (site consulté le 19 avril 2007).

MARTEL, A. (1991). Les droits scolaires des minorités de langue officielle au Canada: de l'instruction à la gestion. Ottawa: Commissariat aux langues officielles.

MARTEL, A. (2002). Droits, écoles et communautés en milieu minoritaire : 1986-2002. Ottawa : Commissariat aux langues officielles.

MINISTÈRE DE L'ÉDUCATION DU NOUVEAU-BRUNSWICK (2007). Les enfants au premier plan.

NOUVEAU-BRUNSWICK, Educational Services Branch, Department of Education (1996). A Resource Guide for Educators of English Second Language Learners, Nouveau-Brunswick. 
PATRICIU, S. et M. Vatz Laaroussi (2002). L'école, sa mission d'intégration des immigrants et la position de ses acteurs en milieu québécois, dans Jeunesses, citoyennetés, violences. Réfugiés albanais en Belgique et au Québec, sous la direction de Michèle Vatz Laaroussi et Altay Manço. Paris : L'Harmattan, coll. «Compétences interculturelles», p.81-111.

POIRIER, C. (2005). Dynamiques et enjeux de la gestion de la diversité ethnique au sein des villes canadiennes, dans Enjeux et défis de l'immigration au NouveauBrunswick: Rendez-vous Immigration, sous la direction de Hélène Destrempes et Paul Ruggieri, p. 343-373.

QUELL, C. 2002. L'immigration et les langues officielles: obstacles et possibilités qui se présentent aux immigrants et aux communautés. Ottawa: Commissariat aux langues officielles.

ROY, J., C. Belkhodja et N. Gallant (2007). Francophone Immigration to Minority Communities: The Challenge of Rural Areas. Nos diverses cités, numéro spécial «Collectivités rurales», sous la direction de Bill Reimer, n 3. p. 87-92.

SASKATCHEWAN LEARNING (1994). Multicultural Education: A Policy Document. http://www.sasked.gov.sk.ca/docs/policy/multi/index.html (site consulté le 2 avril 2007).

SASKATCHEWAN LEARNING (2004). Respect for Diversity. http://www.sasked.gov.sk.ca/branches/curr/evergreen/s_orientation.shtml (site consulté le 19 avril 2007).

SASKATCHEWAN LEARNING (2007). Appendix D - Targeted Funding Support Recognition 2007-08. http://www.sasked.gov.sk.ca/branches/ed_finance/ funding/pdfs/07_08_SectionD.pdf (site consulté le 19 avril 2007). 